\title{
Determination of $\alpha_{\mathrm{s}}$ from hadronic event shapes measured on the $Z^{0}$ resonance
}

\section{L3 Collaboration}

O. Adriani ${ }^{\text {a }}$, M. Aguilar-Benitez ${ }^{\text {b }}$, S. Ahlen ${ }^{c}$, H. Akbari ${ }^{\text {d }}$, J. Alcaraz ${ }^{\text {e }}$, A. Aloisio ${ }^{\text {, }}$, G. Alverson ${ }^{\mathrm{g}}$, M.G. Alviggi ${ }^{f}$, G. Ambrosi ${ }^{h}$, Q. An ${ }^{i}$, H. Anderhub ${ }^{j}$, A.L. Anderson ${ }^{k}$, V.P. Andreev ${ }^{\ell}$,

T. Angelov ${ }^{k}$, L. Antonov ${ }^{\mathrm{m}}$, D. Antreasyan ${ }^{\mathrm{n}}, \mathrm{P}$. Arce ${ }^{\mathrm{b}}, \mathrm{A}$. Arefiev ${ }^{\circ}$, A. Atamanchuk ${ }^{\ell}$,

T. Azemoon ${ }^{\text {p }}$, T. Aziz ${ }^{\mathrm{q}, \mathrm{r}}$, P.V.K.S. Baba ${ }^{\mathrm{i}}$, P. Bagnaia ${ }^{\mathrm{s}}$, J.A. Bakken ${ }^{\mathrm{t}}$, L. Baksay ${ }^{\mathrm{u}}$, R.C. Ball ${ }^{\mathrm{p}}$,

S. Banerjee ${ }^{q}, J$. Bao ${ }^{d}$, R. Barillère ${ }^{\mathrm{e}}$, L. Barone $^{\mathrm{s}}$, R. Battiston ${ }^{\mathrm{h}}, \mathrm{A}$. Bay ${ }^{\mathrm{v}}, \mathrm{F}$. Becattini ${ }^{\mathrm{a}}$,

U. Becker ${ }^{\mathrm{k} j}$, F. Behner ${ }^{\mathrm{j}}$, J. Behrens ${ }^{\mathrm{j}}$, S. Beingessner ${ }^{w}$, Gy.L. Bencze $^{\mathrm{x}}$, J. Berdugo ${ }^{\mathrm{b}}$, P. Berges ${ }^{\mathrm{k}}$,

B. Bertucci ${ }^{\text {h }}$, B.L. Betev ${ }^{\mathrm{m}, \mathrm{j}}$, M. Biasini ${ }^{\mathrm{h}}$, A. Biland ${ }^{\mathrm{j}}$, G.M. Bilei ${ }^{\mathrm{h}}$, R. Bizzarri ${ }^{\mathrm{s}}$, J.J. Blaising ${ }^{\mathrm{w}}$,

P. Blömeke ${ }^{r}$, B. Blumenfeld ${ }^{d}$, G.J. Bobbink ${ }^{\mathrm{e}, y}$, M. Bocciolini ${ }^{\mathrm{a}}$, R. Bock ${ }^{r}$, A. Böhm ${ }^{r}$, B. Borgia ${ }^{\mathrm{s}}$,

D. Bourilkov ${ }^{\mathrm{m}}$, M. Bourquin $^{\mathrm{v}}$, D. Boutigny ${ }^{\mathrm{w}}$, B. Bouwens ${ }^{\mathrm{y}}$, E. Brambilla ${ }^{\mathrm{f}}$, J.G. Branson ${ }^{\mathrm{z}}$,

I.C. Brock $^{\text {aa }}$, M. Brooks ${ }^{\text {ab }}$, C. Buisson ${ }^{\text {ac }}$, A. Bujak ${ }^{\text {ad }}$, J.D. Burger ${ }^{k}$, W.J. Burger ${ }^{v}$, J.P. Burq ${ }^{\text {ac }}$,

J. Busenitz ${ }^{\text {u, X.D. Cai }}{ }^{\mathrm{i}}$, M. Capell ${ }^{\text {ae }}$, M. Caria ${ }^{\text {h }}$, F. Carminati ${ }^{\mathrm{a}}$, A.M. Cartacci ${ }^{\mathrm{a}}$, M. Cerrada ${ }^{\mathrm{b}}$,

F. Cesaroni ${ }^{\text {, }}$ Y.H. Chang ${ }^{k}$, U.K. Chaturvedi ${ }^{1}$, M. Chemarin ${ }^{\text {ac }}$, A. Chen ${ }^{\text {af }}$, C. Chen ${ }^{\text {ag }}$,

G.M. Chen ${ }^{\text {ag }}$, H.F. Chen ${ }^{\text {ah }}$, H.S. Chen ${ }^{\mathrm{ag}}$, J. Chen ${ }^{\mathrm{k}}$, M. Chen ${ }^{\mathrm{k}}$, M.L. Chen ${ }^{\mathrm{p}}$, W.Y. Chen ${ }^{\mathrm{i}}$,

G. Chiefari ${ }^{f}$, C.Y. Chien ${ }^{d}$, M. Chmeissani ${ }^{p}$, S. Chung ${ }^{k}$, C. Civinini ${ }^{a}$, I. Clare ${ }^{k}$, R. Clare ${ }^{k}$,

T.E. Coan ${ }^{a b}$, H.O. Cohn ${ }^{a i}$, G. Coignet ${ }^{w}$, N. Colino ${ }^{\mathrm{e}}$, A. Contin ${ }^{\mathrm{n}}$, F. Crijns ${ }^{\mathrm{aj}}$, X.T. Cui $^{\mathrm{i}}$,

X.Y.Cui ${ }^{i}$, T.S. Dai ${ }^{k}$, R. D'Alessandro ${ }^{a}$, R. de Asmundis ${ }^{f}$, A. Degré ${ }^{w}$, K. Deiters ${ }^{k}$, E. Dénes ${ }^{x}$,

P. Denes ${ }^{t}$, F. DeNotaristefani ${ }^{\mathrm{s}}$, M. Dhina ${ }^{\mathrm{j}}$, D. DiBitonto ${ }^{\mathrm{u}}$, M. Diemoz $^{\mathrm{s}}$, H.R. Dimitrov ${ }^{\mathrm{m}}$,

C. Dionisi ${ }^{\mathrm{s}, \mathrm{e}}$, M.T. Dova ${ }^{\mathrm{i}}$, E. Drago ${ }^{\mathrm{f}}$, T. Driever ${ }^{\mathrm{aj}}$, D. Duchesneau ${ }^{\mathrm{v}}$, P. Duinker ${ }^{\mathrm{y}}$, I. Duran $^{\mathrm{b}}$,

H. El Mamouni ${ }^{\text {ac }}$, A. Engler ${ }^{\text {aa }}$, F.J. Eppling ${ }^{k}$, F.C. Erné ${ }^{y}$, P. Extermann ${ }^{v}$, R. Fabbretti ${ }^{\text {ak }}$,

M. Fabre ${ }^{\text {ak }}$, S. Falciano ${ }^{\text {s }}$, S.J. Fan ${ }^{\text {a }}$, O. Fackler ${ }^{\text {ae }}$, J. Fay ${ }^{\text {ac }}$, M. Felcini ${ }^{\text {e, T. Ferguson }}{ }^{\text {aa }}$,

D. Fernandez ${ }^{b}$, G. Fernandez ${ }^{b}$, F. Ferroni ${ }^{\mathrm{s}}$, H. Fesefeldt ${ }^{\mathrm{r}}$, E. Fiandrini ${ }^{\mathrm{h}}$, J. Field $^{\mathrm{v}}$,

F. Filthaut ${ }^{\text {aj }}$, G. Finocchiaros ${ }^{s}$, P.H. Fisher ${ }^{d}$, G. Forconi ${ }^{v}$, T. Foreman $^{y}$, K. Freudenreich ${ }^{j}$,

W. Friebel ${ }^{\text {am }}$, M. Fukushima ${ }^{k}$, M. Gailloud ${ }^{\text {an }}$, Yu. Galaktionov ${ }^{\text {o.k }}$, E. Gallo ${ }^{\text {a }}$, S.N. Ganguli ${ }^{\mathrm{q}}$,

P. Garcia-Abia ${ }^{\text {b }, ~ S . S . ~ G a u ~}{ }^{\text {af }}$, D. Gele ${ }^{\text {ac }}$, S. Gentile ${ }^{\text {s.e }}$, S. Goldfarb ${ }^{\text {p }}$, Z.F. Gong ${ }^{\text {ah }}$, E. Gonzalez $^{\text {b }}$,

P. Göttlicher ${ }^{r}$, D. Goujon ${ }^{\text {v }, ~ G . ~ G r a t t a ~}{ }^{\text {ao }}$, C. Grinnell ${ }^{k}$, M. Gruenewald ${ }^{\text {ao }}$, C. Gu ${ }^{\text {}}$,

M. Guanziroli ${ }^{i}$, J.K. Guo ${ }^{a}{ }^{\ell}$, V.K. Gupta ${ }^{\mathrm{i}}$, A. Gurtu ${ }^{\mathrm{e}, \mathrm{q}}$, H.R. Gustafson ${ }^{\mathrm{p}}$, L.J. Gutay ${ }^{\text {ad }}$,

K. Hangarter ${ }^{r}$, A. Hasan ${ }^{i}$, D. Hauschildt ${ }^{y}$, C.F. He ${ }^{a \ell}$, T. Hebbeker ${ }^{r}$, M. Hebert ${ }^{z}$, G. Herten ${ }^{k}$,

U. Herten $^{r}$, A. Hervé ${ }^{\mathrm{e}}$, K. Hilgers ${ }^{\mathrm{r}}$, H. Hofer ${ }^{\mathrm{j}}$, H. Hoorani ${ }^{\mathrm{v}}$, G. Hu ${ }^{\mathrm{i}}$, G.Q. Hu ${ }^{\text {at }}$, B. Ille ${ }^{\text {ac }}$,

M.M. Ilyas ${ }^{\mathrm{i}}$, V. Innocente ${ }^{\mathrm{e}, \mathrm{f}}$, H. Janssen ${ }^{\mathrm{e}}$, S. Jezequel ${ }^{\mathrm{w}}$, B.N. Jin ${ }^{\mathrm{ag}}$, L.W. Jones ${ }^{\mathrm{p}}$, A. Kasser ${ }^{\text {an }}$,

R.A. Khan ${ }^{i}$, Yu. Kamyshkov ai, P. Kapinos ${ }^{\ell}$, J.S. Kapustinsky ${ }^{\text {ab }}$, Y. Karyotakis ${ }^{\text {e,w }}$, M. Kaur ${ }^{i}$,

S. Khokhar ${ }^{i}$, M.N. Kienzle-Focacci ${ }^{\mathrm{v}}$, W.W. Kinnison ${ }^{\text {ab }}$, D. Kirkby ${ }^{\text {ao }}$, S. Kirsch ${ }^{\text {am }}$, W. Kittel ${ }^{\text {aj }}$,

A. Klimentov ${ }^{\text {k,o }}$, A.C. König ${ }^{\text {aj, E. Koffeman }}{ }^{y}$, O. Kornadt ${ }^{r}$, V. Koutsenko ${ }^{k, o}$, A. Koulbardis ${ }^{\ell}$,

R.W. Kraemer ${ }^{\text {aa }}$, T. Kramer ${ }^{k}$, V.R. Krastev ${ }^{\text {m,h }}$, W. Krenz ${ }^{\mathrm{r}}$, A. Krivshich ${ }^{\ell}$, J. Krizmanic $^{\mathrm{d}}$,

K.S. Kumar ${ }^{\text {ap }}$, A. Kunin ${ }^{\text {ap.o }}$, G. Landi ${ }^{a}$, D. Lanske ${ }^{\mathrm{r}}$, S. Lanzano ${ }^{\mathrm{f}}$, P. Lebrun ${ }^{\text {ac }}$, P. Lecomte ${ }^{\mathrm{j}}$,

P. Lecoq ${ }^{\mathrm{e}}$, P. Le Coultre ${ }^{j}$, D.M. Lee ${ }^{\mathrm{ab}}$, I. Leedom $^{\mathrm{g}}$, J.M. Le Goff ${ }^{\mathrm{e}}$, R. Leiste ${ }^{\mathrm{am}}$, M. Lenti ${ }^{\mathrm{a}}$,

E. Leonardi s, J. Lettry ${ }^{j}$, X. Leytens ${ }^{y}$, C. Li ${ }^{\text {ah,i }}$, H.T. Li ${ }^{\text {ag }}$, P.J. Li ${ }^{\text {a }}$, X.G. Li ag, J.Y. Liao ${ }^{\text {a }}$,

W.T. Lin ${ }^{\text {af }}$, Z.Y. Lin ah, F.L. Linde ${ }^{\mathrm{e}, \mathrm{y}}$, B. Lindemann ${ }^{\mathrm{r}}$, D. Linnhofer ${ }^{\mathrm{j}}$, Y. Liu ${ }^{\mathrm{i}}$,

W. Lohmann ${ }^{\text {am,e }}$, E. Longo ${ }^{\text {s }}$, Y.S. Lu ${ }^{\text {ag }}$, J.M. Lubbers ${ }^{e}$, K. Lübelsmeyer ${ }^{\mathrm{r}}$, C. Luci ${ }^{\mathrm{s}}$, 
D. Luckey ${ }^{\mathrm{n}, \mathrm{k}}$, L. Ludovici $^{\mathrm{s}}$, L. Luminari ${ }^{\mathrm{s}}$, W.G. Ma ${ }^{\text {ah }}$, M. MacDermott ${ }^{\mathrm{j}}$, P.K. Malhotra ${ }^{\mathrm{q}, 1}$, R. Malik ${ }^{i}$, A. Malinin ${ }^{w, o}$, C. Maña ${ }^{b}$, D.N. Mao ${ }^{p}$, Y.F. Mao ${ }^{a g}$, M. Maolinbay ${ }^{j}$, P. Marchesini ${ }^{j}$, F. Marion ${ }^{\text {w }}$, A. Marin c, J.P. Martin ${ }^{\text {ac }}$, L. Martinez-Laso ${ }^{\text {e }}$, F. Marzano ${ }^{\text {s }}$, G.G.G. Massaro ${ }^{y}$, T. Matsuda $^{k}$, K. Mazumdar ${ }^{\mathrm{q}}$, P. McBride ${ }^{\text {ap }}$, T. McMahon $^{\text {ad }}$, D. McNally ${ }^{\text {j, Th. Meinholz }}{ }^{\text {, }}$, M. Merk ${ }^{\text {aj }}$, L. Merola ${ }^{\mathrm{f}}$, M. Meschini ${ }^{\mathrm{a}}$, W.J. Metzger ${ }^{\text {aj }}$, Y. Mi ${ }^{\mathrm{i}}$, G.B. Mills ${ }^{\text {ab }}$, Y. Mir $^{\mathrm{i}}$, G. Mirabelli ${ }^{\text {s }}$, J. Mnich ${ }^{\mathrm{r}}$, M. Möller ${ }^{\mathrm{r}}$, B. Monteleoni ${ }^{\mathrm{a}}$, R. Morand ${ }^{\mathrm{w}}$, S. Morganti ${ }^{\mathrm{s}}$, N.E. Moulai ${ }^{i}$, R. Mount ${ }^{\text {ao }}$, S. Müller ${ }^{r}$, A. Nadtochy ${ }^{\ell}$, E. Nagy ${ }^{x}$, M. Napolitano ${ }^{f}$, H. Newman ${ }^{\text {ao }}$, C. Neyer ${ }^{j}$, M.A. Niaz ${ }^{i}$, L. Niessen ${ }^{r}$, H. Nowak ${ }^{a m}$, G. Organtini ${ }^{s}$, D. Pandoulas ${ }^{r}$, S. Paoletti ${ }^{a}$, G. Passaleva ${ }^{\text {a,h }}$, S. Patricelli ${ }^{f}$, M. Pauluzzi ${ }^{\text {h }}$, F. Pauss ${ }^{j}$, Y.J. Pei ${ }^{r}$,

D. Perret-Gallix ${ }^{w}$, J. Perrier ${ }^{v}$, A. Pevsner ${ }^{d}$, M. Pieri ${ }^{\text {e.a }}$, P.A. Piroué ${ }^{t}$, F. Plasil ${ }^{\text {ai }}$, V. Plyaskin ${ }^{o}$, M. Pohl ${ }^{j}$, V. Pojidaev ${ }^{0, a}$, N. Produit ${ }^{\mathrm{v}}$, J.M. Qian ${ }^{\mathrm{p}}$, K.N. Qureshi ${ }^{\mathrm{i}}$, R. Raghavan ${ }^{\mathrm{q}}$,

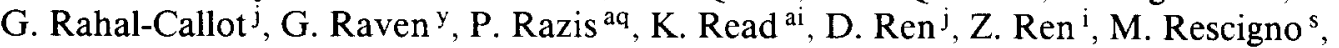
S. Reucroft ${ }^{\mathrm{g}}$, A. Ricker ${ }^{\mathrm{r}}$, S. Riemann ${ }^{\text {am }}$, O. Rind ${ }^{\mathrm{p}}$, H.A. Rizvi ${ }^{i}$, B.P. Roe ${ }^{\mathrm{p}}$, M. Röhner ${ }^{\mathrm{r}}$, S. Röhner ${ }^{r}$, L. Romero ${ }^{b}$, J. Rose $^{r}$, S. Rosier-Lees ${ }^{w}$, R. Rosmalen ${ }^{\text {aj }}$, Ph. Rosselet ${ }^{\text {an }}$, A. Rubbia ${ }^{k}$, J.A. Rubio ${ }^{\text {e,b }}$, H. Rykaczewski ${ }^{j}$, M. Sachwitz $^{\text {am }}$, E. Sajan ${ }^{\text {h, J. Salicio }}{ }^{\text {e,b }}$, J.M. Salicio ${ }^{\text {b }}$, G.S. Sanders ${ }^{\text {ab }}$, A. Santocchia ${ }^{\text {h }}$, M.S. Sarakinos ${ }^{\text {k }}$, G. Sartorelli ${ }^{n, 1}$, M. Sassowsky ${ }^{\text {r }}$, G. Sauvage ${ }^{\text {w }}$, V. Schegelsky ${ }^{\ell}$, K. Schmiemann ${ }^{r}$, D. Schmitz ${ }^{r}$, P. Schmitz ${ }^{r}$, M. Schneegans ${ }^{w}$, H. Schopper ${ }^{a r}$, D.J. Schotanus ${ }^{\text {aj, }}$, S. Shotkin ${ }^{k}$, H.J. Schreiber ${ }^{\text {am }}$, J. Shukla ${ }^{\text {aa }}$, R. Schulte ${ }^{r}$, S. Schulte ${ }^{r}$, K. Schultze ${ }^{r}$, J. Schütte ${ }^{\text {ap }}$, J. Schwenke ${ }^{r}$, G. Schwering ${ }^{r}$, C. Sciacca ${ }^{f}$, I. Scott ${ }^{\text {ap }}$, R. Sehgal ${ }^{i}$, P.G. Seiler ${ }^{\text {ak }}$, J.C. Sens ${ }^{\text {e, } y}$, L. Servoli ${ }^{\text {h }}$, I. Sheer ${ }^{z}$, D.Z. Shen ${ }^{\text {at }}$, S. Shevchenko ${ }^{\text {ao }}$, X.R. Shi ${ }^{\text {ao }}$, E. Shumilov ${ }^{\circ}$, V. Shoutko ${ }^{\circ}$, E. Soderstrom ${ }^{t}$, A. Sopczak ${ }^{z}$, C. Spartiotis ${ }^{d}$, T. Spickermann ${ }^{r}$, P. Spillantini ${ }^{\text {a }}$, R. Starosta ${ }^{\mathrm{r}}$, M. Steuer ${ }^{\mathrm{n}, \mathrm{k}}$, D.P. Stickland ${ }^{\mathrm{t}}$, F. Sticozzi $^{\mathrm{k}}$, H. Stone $^{\mathrm{v}}$,

K. Strauch ${ }^{\text {ap }}$, B.C. Stringfellow ${ }^{\text {ad }}$, K. Sudhakar ${ }^{\text {q,r }}$, G. Sultanov ${ }^{1}$, R.L. Sumner ${ }^{\text {t }}$, L.Z. Sun ${ }^{\text {ah,i }}$, H. Suter ${ }^{j}$, R.B. Sutton aa ${ }^{\text {a J.D. Swain }}{ }^{\mathrm{i}}$, A.A. Syed ${ }^{\mathrm{i}}$, X.W. Tang ${ }^{\mathrm{ag}}$, L. Taylor ${ }^{\mathrm{g}}$, C. Timmermans ${ }^{\text {aj }}$, Samuel C.C. Ting ${ }^{\mathrm{k}}$, S.M. Ting ${ }^{\mathrm{k}}$, M. Tonutti ${ }^{\mathrm{r}}$, S.C. Tonwar ${ }^{\mathrm{q}}$, J. Tóth $^{\mathrm{x}}$, A. Tsaregorodtsev ${ }^{\ell}$, G. Tsipolitis aa , C. Tully ${ }^{\text {ao }}$, K.L. Tung ag, J. Ulbricht ${ }^{\mathrm{j}}$, L. Urbán ${ }^{\mathrm{x}}$, U. Uwer ${ }^{\mathrm{r}}$, E. Valente ${ }^{\mathrm{s}}$, R.T. Van de Walle ${ }^{\text {aj }}$, I. Vetlitsky ${ }^{\circ}$, G. Viertel ${ }^{j}$, P. Vikas ${ }^{i}$, U. Vikas ${ }^{i}$, M. Vivargent ${ }^{w}, H$. Vogel $^{\text {aa }}$, H. Vogt ${ }^{\text {am }}$, I. Vorobiev ${ }^{\circ}$, A.A. Vorobyov ${ }^{\ell}$, L. Vuilleumier ${ }^{\text {an }}$, M. Wadhwa ${ }^{i}$, W. Wallraff ${ }^{r}$, C.R. Wang ${ }^{\text {ah }}$, G.H. Wang ${ }^{\text {aa }}$, J.H. Wang ${ }^{\text {ag }}$, Q.F. Wang ${ }^{\text {ap }}$, X.L. Wang ${ }^{\text {ah }}$, Y.F. Wang ${ }^{\text {a }}$, Z.M. Wang ${ }^{\mathrm{i}, \mathrm{ah}}, \mathrm{A}$. Weber ${ }^{\mathrm{r}}$, J. Weber ${ }^{\mathrm{j}}$, R. Weill ${ }^{\text {an }}$, T.J. Wenaus ${ }^{\text {ae }}$, J. Wenninger $^{\mathrm{v}}$, M. White $^{\mathrm{k}}$, C. Willmott $^{\mathrm{b}}$, F. Wittgenstein ${ }^{\mathrm{e}}$, D. Wright ${ }^{\mathrm{t}}$, R.J. Wu ${ }^{\text {ag }}$, S.X. Wu ${ }^{\mathrm{i}}$, Y.G. Wu ${ }^{\text {ag }}$, B. Wysłouch ${ }^{\mathrm{k}}$, Y.Y. Xie ${ }^{\mathrm{a} \ell}$, Y.D. Xu ${ }^{\mathrm{ag}}$, Z.Z. Xu ${ }^{\text {ah }}$, Z.L. Xue ${ }^{\mathrm{a} \ell}$, D.S. Yan ${ }^{\mathrm{a} \ell}$, X.J. Yan ${ }^{\mathrm{k}}$, B.Z. Yang ${ }^{\mathrm{ah}}$, C.G. Yang ag, G. Yang ${ }^{\mathrm{i}}$, K.S. Yang ${ }^{\mathrm{ag}}$, Q.Y. Yang ${ }^{\mathrm{ag}}$, Z.Q. Yang ${ }^{\mathrm{a} \ell}$, C.H. Ye ${ }^{\mathrm{i}}$, J.B. $^{\mathrm{a}} \mathrm{e}^{\mathrm{ah}}$, Q. Ye ${ }^{\mathrm{i}}$,

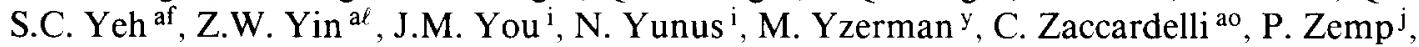
M. Zeng ${ }^{\mathrm{i}}$, Y. Zeng ${ }^{\mathrm{r}}$, D.H. Zhang ${ }^{\mathrm{y}}$, Z.P. Zhang ${ }^{\text {ah, }}$, B. Zhou ${ }^{\mathrm{c}}$, J.F. Zhou ${ }^{\mathrm{r}}$, R.Y. Zhu ${ }^{\text {ao }}$, H.L. Zhuang ${ }^{\mathrm{ag}}$, A. Zichichi ${ }^{\mathrm{n}, \mathrm{e}, \mathrm{i}}$ and B.C.C. van der Zwaan ${ }^{\mathrm{y}}$

\footnotetext{
a INFN - Sezione di Firenze and University of Firenze, I-50125 Florence, Italy

b Centro de Investigaciones Energeticas, Medioambientales y Tecnologicas, CIEMAT, E-28040 Madrid, Spain

c Boston University, Boston, MA 02215, USA

d Johns Hopkins University, Baltimore, MD 21218, USA

e European Laboratory for Particle Physics, CERN, CH-1211 Geneva 23, Switzerland

f INFN - Sezione di Napoli and University of Naples, I-80125 Naples, Italy

$\mathrm{B}$ Northeastern University, Boston, MA 02115, USA

h INFN - Sezione di Perugia and Universitá Degli Studi di Perugia, I-06100 Perugia, Italy

i World Laboratory, FBLJA Project, CH-1211 Geneva, Switzerland

j Eidgenössische Technische Hochschule, ETH Zürich, CH-8093 Zurich, Switzerland

${ }^{k}$ Massachusetts Institute of Technology, Cambridge, MA 02139, USA

'Nuclear Physics Institute, SU-188 350 St. Petersburg, USSR
} 
m Bulgarian Academy of Sciences, Institute of Mechatronics, BG-1113 Sofia, Bulgaria

n INFN - Sezione di Bologna, I-40126 Bologna, Italy

- Institute of Theoretical and Experimental Physics, ITEP, SU-117259 Moscow, USSR

p University of Michigan, Ann Arbor, MI 48109, USA

q Tata Institute of Fundamental Research, Bombay 400005 , India

' $I$. Physikalisches Institut, RWTH, W-5100 Aachen, FRG ${ }^{2}$ and III. Physikalisches Institut, RWTH, W-5I00 Aachen, FRG ${ }^{2}$

s INFN - Sezione di Roma and University of Rome "La Sapienza", I-00185 Rome, Italy

'Princeton University, Princeton, NJ 08544, USA

u University of Alabama, Tuscaloosa, AL 35486, USA

$\checkmark$ University of Geneva, CH-1211 Geneva 4, Switzerland

" Laboratoire de Physique des Particules, LAPP, F-74519 Annecy-le-Vieux, France

$\mathrm{x}$ Central Research Institute for Physics of the Hungarian Academy of Sciences, H-1525 Budapest 114, Hungary

y National Institute for High Energy Physics, NIKHEF, NL-1009 DB Amsterdam, The Netherlands

${ }^{\mathrm{z}}$ University of California, San Diego, CA 92182, USA

aa Carnegie Mellon University, Pittsburgh, PA 15213, USA

ab Los Alamos National Laboratory, Los Alamos, NM 87544, USA

ac Institut de Physique Nucléaire de Lyon, IN2P3-CNRS/Université Claude Bernard. F-69622 Villeurbanne Cedex, France

ad Purdue University, West Lafayette, IN 47907, USA

ae Lawrence Livermore National Laboratory, Livermore, CA 94550, USA

af High Energy Physics Group, Taiwan, ROC

ag Institute of High Energy Physics, IHEP, Beijing, China

ah Chinese University of Science and Technology, Hefei, Anhui 230029 , China

ai Oak Ridge National Laboratory, Oak Ridge, TN 37830, USA

a) University of Nijmegen and NIKHEF, NL-6525 ED Nijmegen, The Netherlands

ak Paul Scherrer Institut, PSI, CH-5232 Villigen, Switzerland

af Shanghai Institute of Ceramics, SIC, Shanghai, China

am Institut für Hochenergiephysik, O-1615 Zeuthen-Berlin, FRG ${ }^{2}$

an University of Lausanne, CH-1015 Lausanne, Switzerland

ao California Institute of Technology, Pasadena, CA 91125, USA

ap Harvard University, Cambridge, MA 02139, USA

aq Department of Natural Sciences, University of Cyprus, Nicosia, Cyprus

ar University of Hamburg, W-2000 Hamburg, FRG

\section{Received 15 April 1992}

We present a study of the global event shape variables thrust and heavy jet mass, of energy-energy correlations and of jet multiplicities based on 250000 hadronic $Z^{0}$ decays. The data are compared to new QCD calculations including resummation of leading and next-to-leading logarithms to all orders. We determine the strong coupling constant $\alpha_{\mathrm{s}}(91.2 \mathrm{GeV})=0.125 \pm 0.003(\exp ) \pm 0.008$ (theor). The first error is the experimental uncertainty. The second error is due to hadronization uncertainties and approximations in the calculations of the higher order corrections.

\section{Introduction}

The strong coupling constant has been determined to second order in $\alpha_{s}$ from hadronic decays of the $Z^{0}$ produced in $\mathrm{e}^{+} \mathrm{e}^{-}$collisions using measurements of jet multiplicities [1-5], energy-energy correlations [6-

\section{Deceased.}

2 Supported by the German Bundesministerium für Forschung und Technologie.
$8,4]$ and other event shape variables $[3,8,4,5]$. The dominant error is due to contributions beyond second order in the perturbative expansion, which are not included in the corresponding QCD calculations.

Recently new calculations of hadronic event shapes including the second order results and the resummation of leading and next-to-leading logarithms to all orders in QCD perturbation theory have been made [9-11]. Therefore the theoretical uncertainties due to uncalculated terms are expected to be reduced with 
respect to second order calculations. The new computations are available for the global event shape variables thrust [12] and heavy jet mass [13] and for energy-energy correlations [14] in the back-to-back region. Also the average jet multiplicity calculated in the new $k_{\perp}$ jet clustering scheme [15] has been computed [16] however certain terms have not yet been included in the resummation [17]

We report here on measurements of those four event shape variables at the $Z^{0}$ resonance using the $L 3$ detector at LEP. Comparing our data to the predictions of the new QCD calculations we derive values for the strong coupling constant $\alpha_{\mathrm{s}}$ at $\sqrt{s}=m_{\mathrm{Z}}$.

\section{The $\mathbf{L} 3$ detector}

The L3 detector covers $99 \%$ of $4 \pi$ [18]. The detector consists of a central tracking chamber, a high resolution electromagnetic calorimeter composed of bismuth germanium oxide crystals, a ring of scintillation counters, a uranium and brass hadron calorimeter with proportional wire chamber readout, and an accurate muon chamber system. These detectors are installed in a $12 \mathrm{~m}$ diameter magnet which provides a uniform field of $0.5 \mathrm{~T}$ along the beam direction.

For the present analysis, we use data collected in the following ranges of polar angle:

- $40^{\circ}<\theta<140^{\circ}$ for the tracking chamber,

$-11^{\circ}<\theta<169^{\circ}$ for the electromagnetic calorimeter,

$-5^{\circ}<\theta<175^{\circ}$ for the hadron calorimeter,

- $36^{\circ}<\theta<144^{\circ}$ for the muon chambers.

\section{Selection of hadronic events}

Events collected at a center of mass energy of $\sqrt{s}=$ 91.2 GeV from the 1991 LEP running period, corresponding to an integrated luminosity of about $8 \mathrm{pb}^{-1}$. are used for this analysis.

The primary trigger for hadronic events requires a total energy of about $15 \mathrm{GeV}$ in the calorimeters. This trigger is in logical $O R$ with a trigger using the barrel scintillation counters and with a charged track trigger. The combined trigger efficiency for selected hadronic events exceeds $99.9 \%$.
Events of type $\mathrm{e}^{+} \mathrm{e}^{-} \rightarrow$ hadrons are selected and analyzed by two independent methods: one is based on the energy measured in the electromagnetic and hadronic calorimeters, the other one uses charged tracks measured in the tracking chamber. The results presented in this paper are based on the calorimeter method, which is the more precise one. The track measurements are used to cross check the first method and to estimate the experimental errors.

For the calorimeter based selection, we require:

$-N_{\text {cluster }}>12$,

- $0.6<E / \sqrt{s}<1.4$,

- $\left|E_{\|}\right| / E<0.4, \quad E_{\perp} / E<0.4$,

where $E$ is the total energy observed in the calorimeters, $E_{\|}$is the energy imbalance along the beam direction, $E_{\perp}$ is the energy imbalance in the plane perpendicular to the beam direction, and $N_{\text {cluster }}$ is the number of calorimetric clusters with energy greater than $100 \mathrm{MeV}$. We select 248100 hadronic events.

The selection based on charged tracks is described in detail in ref. [19]. We require at least 5 tracks originating close to the interaction point, each with a transverse momentum of at least $100 \mathrm{MeV}$ with respect to the beam axis. A cut on the polar angle of the thrust axis of $\left|\cos \theta_{\text {thrust }}\right|<0.7$ ensures that the selected events are well contained in the tracking chamber. With these criteria we find 169700 hadronic $Z^{0}$ decays.

Monte Carlo studies show that the efficiencies of the two selections are $98.5 \%$ and $66.7 \%$ respectively. The low efficiency for the second method is due to the $\cos \theta_{\text {thrust }}$ cut. The main background sources are $\tau^{+} \tau^{-}$ and $\mathrm{e}^{+} \mathrm{e}^{-}+$hadrons final states. Applying the same cuts to background Monte Carlo events, the contaminations in the selected $\mathrm{e}^{+} \mathrm{e}^{-} \rightarrow$ hadrons samples are estimated to be less than $0.2 \%$ for the first selection, and to be less than $0.1 \%$ for the second selection.

Monte Carlo events were generated by the parton shower programs JETSET 7.3 [20] and HERWIG 5.3 [21] and passed through the L3 detector simulation [22].

\section{Definition of variables}

It is important to consider all the observables for which the new QCD calculations are available. Since they are affected differently by higher order effects 
and hadronization corrections, a comparison of the corresponding $\alpha_{\mathrm{s}}$ values allows an estimation of the size of theoretical uncertainties.

The global event shape variable thrust $T[12]$ is defined as

$T=\max \frac{\sum\left|\boldsymbol{p}_{i} \cdot \boldsymbol{n}_{\mathrm{T}}\right|}{\sum\left|\boldsymbol{p}_{i}\right|}$,

where $\boldsymbol{p}_{i}$ is the momentum vector of the hadron $i$. The thrust axis $n_{\Upsilon}$ is determined by maximizing the above expression. The thrust values can vary between 0.5 and 1.

The heavy jet mass $M_{\mathrm{H}}$ is defined as [13]

$M_{\mathrm{H}}=\max \left[M_{1}\left(\boldsymbol{n}_{\mathrm{T}}\right), M_{2}\left(\boldsymbol{n}_{\mathrm{T}}\right)\right]$,

where $M_{1,2}$ are the invariant masses in the two hemispheres defined by the thrust axis:

$M_{l}^{2}=\left(\sum_{\text {hemisphere } l} p_{i}\right)^{2}$

where $p_{i}$ is the four momentum of particle $i$. Here we use the normalized quantity

$\rho=M_{\mathrm{H}}^{2} / s$.

The energy-energy correlation (EEC) [14] can be defined as a histogram of all angles between any particles $i, j$ in hadronic events weighted with the product of their energies, and averaged over $N$ events:

\section{$\operatorname{EEC}\left(\chi_{\text {bin }}\right)$}

$$
=\frac{1}{\Delta_{\text {bin }} \cdot N} \sum_{\text {events }}^{N} \sum_{i, j} \frac{E_{i} \cdot E_{j}}{s} \delta_{\text {bin }}\left(\chi_{\text {bin }}-\chi_{i j}\right) .
$$

$\delta_{\text {bin }}\left(\chi_{\text {bin }}-\chi_{i j}\right)$ is 1 for angles $\chi_{i j}$ inside the bin around $\chi_{\text {bin }}$ and 0 otherwise. $\Delta_{\text {bin }}$ denotes the bin width. For 2-jet events most angles are close to $0^{\circ}$ or $180^{\circ}$, while events with hard gluon radiation contribute to the central region. To determine $\alpha_{s}$ we use here the back-toback region with $\chi \geqslant 90^{\circ}$, for which the new calculations have been performed [11].

Jets are reconstructed using the new " $k_{\perp}$ " algorithm [15]. For each pair of particles $i$ and $j$ the expression

$y_{i j}=2\left[\min \left(E_{i}^{2}, E_{j}^{2}\right) / s\right]\left(1-\cos \theta_{i j}\right)$ is evaluated. $E_{i}$ and $E_{j}$ are the energies and $\theta_{i j}$ is the angle between particles $i$ and $j$. The pair for which $y_{i j}$ is smallest is replaced by a pseudoparticle $l$ with four-momentum

$p_{l}=p_{i}+p_{j}$.

This procedure is repeated until all $y_{i j}$ exceed the jet resolution parameter $y_{\text {cut }}$. The remaining (pseudo)particles are called jets. Increasing $y_{\text {cut }}$ lowers the fraction of multijet events but increases the separation of the jets.

The difference between the $k_{\perp}$ and the JADE jet algorithms [23], which we used previously [1] lies in the definition of $y_{i j}$. While in the JADE scheme the scaled invariant mass squared is used, the quantity $y_{i j}$ in the new algorithm corresponds to the scaled relative transverse momentum $k_{\perp}$ squared. Here we use the $k_{\perp}$ jet algorithm, since in this scheme (but not in the JADE scheme) the resummation of the leading and next-to-leading terms is possible [24,25,16].

In this paper we measure the average jet multiplicity

$\left\langle n_{\mathrm{jet}}\right\rangle=\frac{1}{N} \sum_{i=1}^{N} n_{\mathrm{jet}}^{i}$

as a function of $y_{\text {cut }}$. Here $N$ is the total number of events.

\section{Measurement of event shape variables}

The four distributions $T, \rho$, EEC and $\left\langle n_{\text {jet }}\right\rangle$ are measured separately from calorimetric clusters and charged tracks. In the first case energies and in the second case particle momenta are used. The particle masses are assumed to be zero. In the above definitions we replace the center of mass energy $\sqrt{s}$ by the measured sum of all energies or momenta, respectively.

We measure the event shape distributions $T, \rho, \mathrm{EEC}$ and the jet fractions $f_{i}=\sigma_{i-\text { jets }} / \sigma_{\text {tot }}$ in the form of histograms. These distributions are corrected for detector effects, acceptance and resolution, and initial and final state photon radiation. The average jet multiplicity $\left\langle n_{\mathrm{jet}}\right\rangle$ is then calculated from the jet fractions $f_{i}$. We choose the histogram bin widths such that they are bigger than the experimental resolution by a factor of at least 1.5. The detector resolution effects for the 


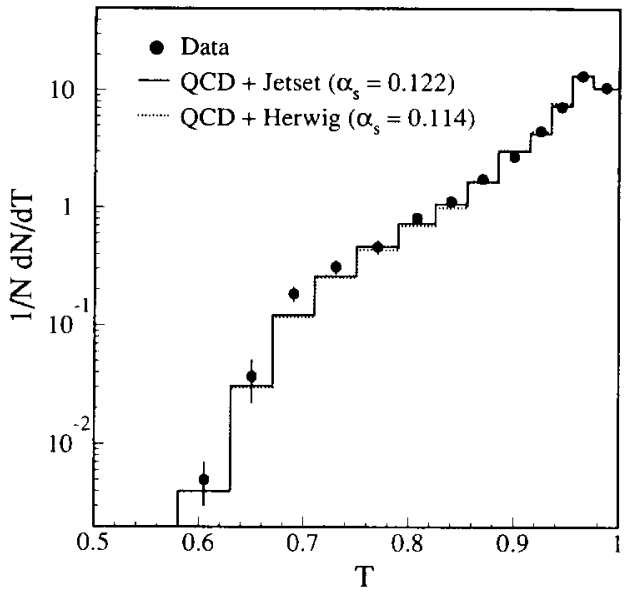

Fig. 1. Measured thrust distribution in comparison with the QCD predictions. The theoretical curves are calculated for a scale $\mu=91.2 \mathrm{GeV}$ and the fitted $\alpha_{\mathrm{s}}\left(m_{\mathrm{Z}}\right)$ values as shown in the figure. The solid (dashed) line is obtained using JETSET (HERWIG) for the hadronization correction.

observables thrust and scaled heavy jet mass squared are corrected using the regularized unfolding program [25], as described in ref. [19]. The EEC distribution is corrected bin by bin [6]. The jet fractions are determined using a matrix unfolding procedure [1]. The resolution function is given implicitly by Monte Carlo events. They are generated by the programs JETSET 7.3 and HERWIG 5.3 and include the full detector simulation. To correct for detector acceptance and in particular for the cut in $\cos \theta_{\text {thrust }}$, we apply a binby-bin correction. Similarly we correct for initial and final state radiation. The systematic errors on each unfolded data point are due to uncertainties in the detector simulation and biases from the Monte Carlo program used for the unfolding. Statistical uncertainties are negligible. The sizes of the experimental errors are estimated by

- varying the energy response of the calorimeters in the Monte Carlo simulation,

- analyzing the difference in the unfolded distributions using the programs JETSET and HERWIG,

- comparing the corrected distributions obtained from calorimetric clusters and charged tracks.

The experimental uncertainties are typically a few percent per histogram bin.

The corrected distributions of the observables $T$

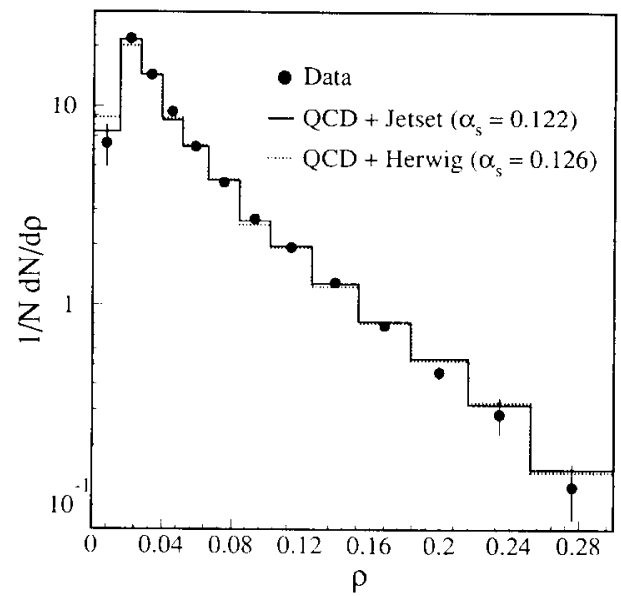

Fig. 2. Measured distribution of the variable scaled heavy jet mass squared $\rho$ in comparison with the QCD predictions. The theoretical curves are calculated for a scale $\mu=91.2$ $\mathrm{GeV}$ and the fitted $\alpha_{\mathrm{S}}\left(m_{\mathrm{Z}}\right)$ values as shown in the figure. The solid (dashed) line is obtained using JETSET (HERWIG) for the hadronization correction.

and $\rho$ are shown in figs. 1 and 2 and given in tabular form in ref. [19]. The EEC distribution is shown in fig. 3 (back-to-back region only) and given in table 1. It agrees within errors with our previous measurement [6], which covered only the central region between $25.2^{\circ}$ and $154.8^{\circ}$. The average jet multiplicities as function of $y_{\text {cut }}$ are listed in table 2 and are shown in fig. 4 .

\section{QCD predictions}

The QCD predictions in fixed order perturbation theory can not take into account the effect of multiple gluon emission. In second order calculations two gluons can be emitted at most. For variables like thrust, heavy jet mass etc. this leads to a singular behavior of the distributions in kinematic regions where multigluon emission becomes dominant. This is a direct consequence of the collinear and infrared divergence of the gluon emission cross section. It is possible to isolate the singular terms in every order of perturbation theory and to sum them up in the form of an exponential series. One thus obtains a meaningful result for all regions of phase space. In the calculations described in refs. [9-11] and used for this analysis, all 
Table 1

Measured EEC distribution corrected for detector effects and photon radiation. The errors are the combined statistical and systematic uncertainties.

\begin{tabular}{lccc}
\hline$\chi$ & EEC $(\chi)$ & $\chi$ & EEC $(\chi)$ \\
\hline $0.0^{\circ}-3.6^{\circ}$ & $2.21 \pm 0.10$ & $176.4^{\circ}-180.0^{\circ}$ & $0.80 \pm 0.02$ \\
$3.6^{\circ}-9.0^{\circ}$ & $1.15 \pm 0.06$ & $171.0^{\circ}-176.4^{\circ}$ & $1.27 \pm 0.04$ \\
$9.0^{\circ}-14.4^{\circ}$ & $0.66 \pm 0.02$ & $165.6^{\circ}-171.0^{\circ}$ & $0.90 \pm 0.03$ \\
$14.4^{\circ}-19.8^{\circ}$ & $0.41 \pm 0.01$ & $160.2^{\circ}-165.6^{\circ}$ & $0.60 \pm 0.02$ \\
$19.8^{\circ}-25.2^{\circ}$ & $0.272 \pm 0.008$ & $154.8^{\circ}-160.2^{\circ}$ & $0.418 \pm 0.013$ \\
$25.2^{\circ}-30.6^{\circ}$ & $0.201 \pm 0.006$ & $149.4^{\circ}-154.8^{\circ}$ & $0.307 \pm 0.009$ \\
$30.6^{\circ}-36.0^{\circ}$ & $0.159 \pm 0.005$ & $144.0^{\circ}-149.4^{\circ}$ & $0.237 \pm 0.007$ \\
$36.0^{\circ}-41.4^{\circ}$ & $0.133 \pm 0.005$ & $138.6^{\circ}-144.0^{\circ}$ & $0.190 \pm 0.006$ \\
$41.4^{\circ}-46.8^{\circ}$ & $0.115 \pm 0.004$ & $133.2^{\circ}-138.6^{\circ}$ & $0.157 \pm 0.005$ \\
$46.8^{\circ}-52.2^{\circ}$ & $0.102 \pm 0.003$ & $127.8^{\circ}-133.2^{\circ}$ & $0.134 \pm 0.004$ \\
$52.2^{\circ}-57.6^{\circ}$ & $0.093 \pm 0.003$ & $122.4^{\circ}-127.8^{\circ}$ & $0.117 \pm 0.004$ \\
$57.6^{\circ}-63.0^{\circ}$ & $0.086 \pm 0.003$ & $117.0^{\circ}-122.4^{\circ}$ & $0.104 \pm 0.003$ \\
$63.0^{\circ}-68.4^{\circ}$ & $0.081 \pm 0.002$ & $111.6^{\circ}-117.0^{\circ}$ & $0.095 \pm 0.003$ \\
$68.4^{\circ}-73.8^{\circ}$ & $0.078 \pm 0.002$ & $106.2^{\circ}-111.6^{\circ}$ & $0.088 \pm 0.003$ \\
$73.8^{\circ}-79.2^{\circ}$ & $0.076 \pm 0.003$ & $100.8^{\circ}-106.2^{\circ}$ & $0.083 \pm 0.003$ \\
$79.2^{\circ}-84.6^{\circ}$ & $0.075 \pm 0.002$ & $95.4^{\circ}-100.8^{\circ}$ & $0.079 \pm 0.003$ \\
$84.6^{\circ}-90.0^{\circ}$ & $0.075 \pm 0.002$ & $90.0^{\circ}-95.4^{\circ}$ & $0.076 \pm 0.002$
\end{tabular}

Table 2

Measured average jet multiplicities for the $k_{\perp}$ algorithm. All numbers are corrected for detector effects and photon radiation. The errors are the combined statistical and systematic uncertainties.

\begin{tabular}{lc}
\hline$y_{\text {cut }}$ & $\left\langle n_{\text {jet }}\right\rangle$ \\
\hline 0.001 & $3.93 \pm 0.07$ \\
0.002 & $3.20 \pm 0.03$ \\
0.004 & $2.75 \pm 0.018$ \\
0.006 & $2.581 \pm 0.013$ \\
0.008 & $2.492 \pm 0.010$ \\
0.010 & $2.428 \pm 0.009$ \\
0.020 & $2.269 \pm 0.006$ \\
0.040 & $2.153 \pm 0.003$ \\
0.060 & $2.102 \pm 0.003$ \\
0.090 & $2.071 \pm 0.003$ \\
0.100 & $2.053 \pm 0.003$ \\
0.120 & $2.038 \pm 0.003$ \\
0.140 & $2.028 \pm 0.002$ \\
0.160 & $2.021 \pm 0.002$ \\
0.180 & $2.016 \pm 0.001$ \\
0.200 & $2.012 \pm 0.001$ \\
0.220 & $2.0077 \pm 0.0010$ \\
0.240 & $2.0052 \pm 0.0010$ \\
0.260 & $2.0029 \pm 0.0010$ \\
0.280 & $2.0016 \pm 0.0006$ \\
\hline
\end{tabular}

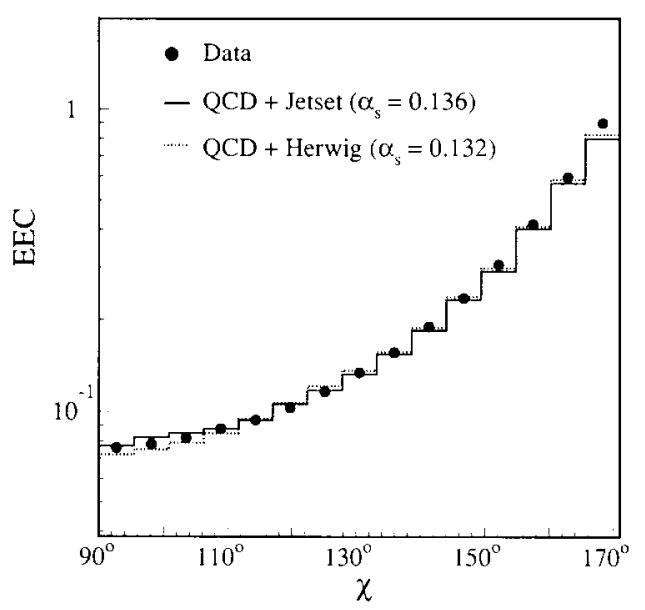

Fig. 3. Measured energy-energy correlation function EEC in comparison with the QCD predictions. The theoretical curves are calculated for a scale $\mu=91.2 \mathrm{GeV}$ and the fitted $\alpha_{\mathrm{S}}\left(m_{\mathrm{Z}}\right)$ values as shown in the figure. The solid (dashed) line is obtained using JETSET (HERWIG) for the hadronization correction.

second order terms [26,27] are included together with leading and next-to-leading corrections to all orders. These corrections are of the form $\alpha_{\mathrm{s}}^{n} \ln ^{m} y$ with $n \geqslant 1$ and $m \geqslant n$. Subleading terms of the form $\alpha_{\mathrm{s}}^{n} \ln ^{m} y$ with $m<n$ are not included beyond second order 


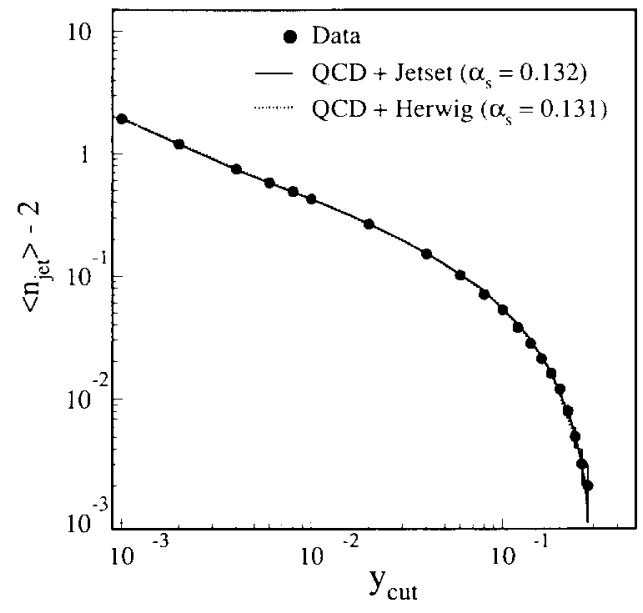

Fig. 4. Measured distribution of the average jet multiplicity $\left\langle n_{\text {jet }}\right\rangle$ as a function of $y_{\text {cut }}$ in comparison with the QCD predictions. The theoretical curves are calculated for a scale $\mu=91.2 \mathrm{GeV}$ and the fitted $c_{\mathrm{S}}\left(m_{\mathrm{Z}}\right)$ values as shown in the figure. The solid (dashed) line is obtained using JETSET (HERWIG) for the hadronization correction.

$(n>2)$. Here $y$ stands for $1-T, \rho,(1+\cos \chi) / 2$ and $y_{\mathrm{cut}}$, respectively. In the 2-jet region $y$ is small and the corrections large. An important improvement of the new QCD calculations with respect to the second order formulas is their ability to describe also the low $y$ region. The authors of refs. [9-11,16] have provided us with computer programs which we used for the QCD calculations.

The calculations for the distributions of the four variables $V\left(=T, \rho, \chi, y_{\text {cut }}\right)$ are given in the form of analytical functions

$f^{\text {pert }}\left(V ; s, \alpha_{\mathrm{s}}(\mu), \mu\right)$.

The strong coupling constant $\alpha_{\mathrm{s}}$ is evaluated at the renormalization scale $\mu$. To translate the value $\alpha_{\mathrm{s}}(\mu)$ into $\alpha_{\mathrm{S}}\left(m_{\mathrm{Z}}\right)$ we use the relation given in ref. [28] for 5 quarks. The QCD calculations contain terms to all orders in $\alpha_{\mathrm{s}}$, and $\mu=\sqrt{s}$ is expected to be a good choice of the renormalization scale $[9-11,16]$. We estimate the uncertainty due to the approximations made in the calculations of the terms beyond second order by a variation of the scale between $0.5 \sqrt{s}$ and $2 \sqrt{s}$. This leads to an estimated uncertainty in $\alpha_{\mathrm{s}}$ of about $\Delta \alpha_{5}= \pm 0.005$.

To study the effects of hadronization and decays we use the parton shower programs JETSET 7.3 and
HERWIG 5.4 with string and cluster fragmentation, respectively. The fragmentation parameters are determined from a comparison of predicted and measured distributions for several event shape variables $[19,29]$. Both generators are found to give a good description of our measurements. We fold the perturbative calculations for $T$ and $\rho$ with the probability $p^{\text {non-pert }}\left(V^{\prime}, V\right)$ to find a value $V$ after fragmentation and decays for a parton level value $V^{\prime}$ :

$f(V)=\int f^{\text {pert }}\left(V^{\prime}\right) \cdot p^{\text {non-pert }}\left(V^{\prime}, V\right) \mathrm{d} V^{\prime}$.

For the EEC distribution and the average jet multiplicity we apply a bin-by-bin hadronization correction. We compare the resulting differential cross section $f(V)$ to our measurements. The correction for hadronization and decays changes the perturbative prediction by less than $5 \%$ for the distributions thrust and scaled heavy jet mass squared, with the exception of the regions $T>0.93$ and $\rho<0.07$, where the effect reaches $15 \%$. The EEC distribution is modified by $5 \%-15 \%$. The quantity $\left\langle n_{\text {jet }}\right\rangle-2$ is modified by less than $5 \%$.

We estimate the uncertainties in the probabilities $p^{\text {non-pert }}\left(V^{\prime}, V\right)$ and the corresponding error in $\alpha_{\mathrm{s}}$ by - a change of fragmentation parameters in the JETSET model within their errors [19],

- a variation of the parton shower cutoff $Q_{0}$ between $1 \mathrm{GeV}$ and $2 \mathrm{GeV}$ in the JETSET program and of the fragmentation parameters accordingly [19],

- a comparison between the predictions of the two models JETSET and HERWIG.

The uncertainties estimated with the first two methods are found to be small $\left(\Delta x_{s}= \pm 0.002\right)$ compared to the differences between HERWIG and JETSET. Those correspond to differences in the measured strong coupling constants of up to 0.008 .

The effect of quark masses, in particular for $b \bar{b}$ events, which is not included in the QCD calculations, is found to be negligible for the measurement of $\alpha_{\mathrm{s}}$ [30].

\section{Results}

In order to derive $\alpha_{\mathrm{S}}\left(m_{\mathrm{Z}}\right)$, we fit the theoretical distribution $f(V)$ to the measured event shape distributions for a fixed scale $\mu=\sqrt{s}$. For the fit we use 
Table 3

Central values and errors of $\alpha_{\mathrm{S}}\left(m_{Z}\right)$ derived from the event shape variables thrust, heavy jet mass, EEC and average jet multiplicity.

\begin{tabular}{llllll}
\hline Observable & Fit range & $\alpha_{\mathrm{s}}\left(m_{\mathrm{Z}}\right)$ & Exp. err. & Hadr. unc. & Scale unc. \\
\hline thrust & $0.71 \leqslant T \leqslant 1.0$ & 0.118 & \pm 0.004 & \pm 0.004 & +0.007 \\
jet mass & $0.0 \leqslant \rho \leqslant 0.3$ & 0.124 & \pm 0.003 & \pm 0.003 & +0.006 \\
EEC & $90^{\circ} \leqslant \chi \leqslant 171^{\circ}$ & 0.134 & \pm 0.003 & \pm 0.003 & +0.004 \\
$\left\langle n_{\text {jet }}\right\rangle$ & $0.002 \leqslant y_{\text {cut }} \leqslant 0.1$ & 0.132 & \pm 0.003 & \pm 0.002 & +0.006 \\
\end{tabular}

the ranges as given in table 3 . In case of the variables $T$ and $\rho$ they correspond to regions dominated by 2 and 3 -jet production $(y<0.3)$. The $\chi$ range corresponds to the region for which the new EEC calculations are available. Since the average jet multiplicities at neighboring $y_{\text {cut }}$ values are strongly correlated, we have included in the fit only the points at $y_{\text {cut }}=0.002$, 0.02 and 0.10 . We later compare data and QCD calculations for values of $y_{\text {cut }}$ above 0.001 , corresponding to a transverse momentum $k_{\perp}$ of about $3 \mathrm{GeV}$. For smaller values of the jet resolution parameter the effects of hadronization and decays become very large. The values of $\alpha_{s}\left(m_{Z}\right)$ derived from the four event shape variables together with their systematic errors are shown in table 3. Figs. 1 to 4 show the QCD fits in comparison with the experimental data. Two curves are shown, corresponding to the hadronization correction using JETSET and HERWIG and the fitted $\alpha_{\mathrm{S}}$ values. In all cases a good fit is obtained.

The results in table 3 are the mean values of the two $\alpha_{\mathrm{S}}$ values obtained with JETSET and HERWIG fragmentation correction. The hadronization error is taken to be the quadratic average of half the difference of those two $\alpha_{5}$ values and the uncertainties estimated by changing fragmentation parameters and the parton shower cutoff inside JETSET as described above. The experimental uncertainties in table 3 are determined by repeating the $\alpha_{\mathrm{s}}$ determination for different energy response factors and unfolding methods, and by using tracks instead of calorimetric clusters. The scale error is obtained by repeating the $\alpha_{5}$ fit for different values of the renormalization scale in the interval $0.5 \sqrt{s} \leqslant$ $\mu \leqslant 2 \sqrt{s}$. For all these scales a good fit is obtained except for the energy-energy correlations, which give a large $\chi^{2}$ value for scales below $\sqrt{s}$. To investigate the dependence of our results on the fit range we have omitted up to two bins at either end of the interval and repeated the fit. In all cases the results differ from the $\alpha_{\mathrm{s}}$ values quoted in table 3 by at most 0.001 .

The values for the strong coupling constant derived from thrust and heavy jet mass are consistent with those reported in refs. $[9,5,31]$, The $\alpha_{\mathrm{s}}$ value obtained from the average jet multiplicity agrees within errors with the strong coupling constant measured from the differential 2-jet rate [31].

The $\alpha_{\mathrm{s}}$ values from the distributions thrust, heavy jet mass and energy-energy correlations are affected differently by higher order corrections and hadronization effects. To obtain a combined value for the strong coupling constant we take the unweighted average of the first three $\alpha_{\mathrm{s}}$ values of table 3 and obtain $\alpha_{\mathrm{s}}=$ $0.125 \pm 0.003(\mathrm{exp})$. Since in the calculation of the average jet multiplicity some terms are missing, we prefer not to include the last $\alpha_{\mathrm{s}}$ value in table 3 in our combined result [17].

We estimate the total theoretical error, due to both the approximations in the higher order corrections and hadronization effects, independently

- from the quadratically combined errors for hadronization and scale dependence in table 3, resulting in approximately $\Delta \alpha_{\mathrm{s}}= \pm 0.006$,

- and from the spread of the three $\alpha_{\mathrm{s}}$ values from thrust, heavy jet mass and EEC, yielding \pm 0.008 .

The two estimates agree well. We conservatively assign the larger one as the theoretical uncertainty in our $\alpha_{\mathrm{s}}$ determination.

The combined result is therefore

$\alpha_{S}\left(m_{Z}\right)=0.125 \pm 0.003(\exp ) \pm 0.008$ (theor).

This result agrees within errors with the values of the strong coupling constant which we measured pre- 
viously ${ }^{\# 1}$. The $\alpha_{\mathrm{s}}$ value of 0.125 also agrees with our measurement of $\alpha_{\mathrm{S}}=0.134 \pm 0.030$ from an analysis of the partial hadronic $Z^{0}$ width [32].

\section{Summary and conclusions}

We have determined the strong coupling constant with a precision of 7\% from hadronic event shapes measured on the $Z^{0}$ resonance using new QCD calculations including exponentiation of leading and nextto-leading terms. We obtain

$$
\chi_{\mathrm{s}}(91.2 \mathrm{GeV})
$$

$$
=0.125 \pm 0.003(\exp ) \pm 0.008 \text { (theor) } \text {. }
$$

The error is dominated by theoretical uncertainties due to approximations in the perturbative calculations and hadronization effects. This error is estimated from the spread of the $\alpha_{\mathrm{s}}$ values obtained from the shape variables thrust, heavy jet mass and energyenergy correlations. Based on the results in table 3 we arrive at an estimate of the theoretical error which is larger than the estimate of about \pm 0.004 in refs. [5,31]. A better determination of the theoretical uncertainties will become possible when the new calculation techniques have been applied to several more event shape variables.

\section{Acknowledgement}

We wish to express our gratitude to the CERN accelerator divisions for the excellent performance of the LEP machine. We acknowledge the effort of all engineers and technicians who have participated in the construction and maintenance of this experiment. We are grateful to S. Catani, G. Turnock and B.R. Webber for many useful discussions and for providing us with their computer programs.

\# We found $\alpha_{\mathrm{s}}=0.116 \pm 0.011$ from jet production [1], $0.122 \pm 0.011$ from energy-energy correlations and $0.115 \pm 0.009$ from the asymmetry of energy correlations [6]. The average of these numbers is $0.118 \pm 0.010$.

\section{References}

[1] L3 Collab., B. Adeva et al., Phys. Lett. B 248 (1990) 464.

[2] MARK II Collab., S. Komamiya et al., Phys. Rev. Lett. 64 (1990) 987;

OPAL Collab., M.Z. Akrawy et al., Phys. Lett. B 235 (1990) 389;

DELPHI Collab., P. Abreu et al., Phys. Lett. B 247 (1990) 167;

OPAL Collab., M.Z. Akrawy et al., Z. Phys. C 49 (1991) 375

[3] ALEPH Collab., D. Decamp et al., Phys. Lett. B 255 (1991) 623 .

[4] DELPHI Collab., P. Abreu et al., Determination of $\alpha_{s}$ in second order QCD in hadronic $Z^{0}$ decays, CERN preprint CERN-PPE/91-181 (revised version).

[5] OPAL Collab., P.D. Acton et al, A Global Determination of $\alpha_{\mathrm{S}}\left(M_{\mathrm{Z}^{0}}\right)$ at LEP, CERN preprint CERN-PPE/92-18.

[6] L3 Collab., B. Adeva et al., Phys. Lett. B 257 (1991) 469.

[7] OPAL Collab., M.Z. Akrawy et al., Phys. Lett. B 252 (1990) 159;

DELPHI Collab., P. Abreu et al., Phys. Lett. B 252 (1990) 149;

OPAL Collab., P.D. Acton et al., Phys. Lett. B 276 (1992) 547.

[8] ALEPH Collab., D. Decamp et al., Phys. Lett. B 257 (1991) 479 .

[9] S. Catani et al., Phys. Lett. B 263 (1991) 491.

[10] S. Catani, G. Turnock and B.R. Webber, Phys. Lett. 272 (1991) 368.

[11] J.C. Collins and D.E. Soper, Nucl. Phys. B 193 (1981) 381; B 197 (1982) 446; B 284 (1987) 253;

J. Kodaira and L. Trentadue, Phys. Lett. B 123 (1983) 335 ;

G. Turnock, Energy-energy correlation distribution in $\mathrm{e}^{+} \mathrm{e}^{-}$annihilation, Cavendish preprint CavendishHEP-92/3 (1992).

[12] S. Brandt et al., Phys. Lett. 12 (1964) 57;

E. Farhi, Phys. Rev. Lett. 39 (1977) 1587.

[13] L. Clavelli, Phys. Lett. B 85 (1979) 111;

MARK II Collab., A. Peterson et al., Phys. Rev. D 37 (1988) 1:

TASSO Collab., W. Braunschweig et al,, Z. Phys. C 45 (1989) 11.

[14] C.L. Basham et al., Phys. Rev. Lett. 41 (1978) 1585; Phys. Rev. D 17 (1978) 2298; D 19 (1979) 2018; D 24 (1981) 2382.

[15] Y.L. Dokshitzer, Contribution to the Workshop on Jets at LEP and HERA, Durham (1990);

N. Brown and W.J. Stirling, Finding jets and summing soft gluons: a new algorithm, Rutherford preprint RAL91-049;

S. Catani et al., Phys. Lett. B 269 (1991) 432;

S. Bethke et al., Nucl. Phys. B 370 (1992) 310 
[16] S. Catani et al., Average number of jets in $\mathrm{e}^{+} \mathrm{e}^{-}$ annihilation, CERN preprint CERN-TH.6328/91 (revised version).

[17] S. Catani and B.R. Webber, private communication.

[18] L3 Collab., B. Adeva et al., Nucl. Instrum. Methods A 289 (1990) 35 .

[19] L3 Collab., B. Adeva et al., Studies of hadronic event structure and comparisons with QCD models at the $Z^{0}$ resonance, CERN preprint CERN-PPE/92-50, to be published in Z. Phys. C.

[20] JETSET Monte Carlo Program:

T. Sjöstrand, Comput. Phys. Commun. 39 (1986) 347 ;

T. Sjöstrand and M. Bengtsson, Comput. Phys. Commun. 43 (1987) 367.

[21] HERWIG Monte Carlo Program:

G. Marchesini and B.R. Webber, Nucl. Phys. B 310 (1988) 461;

I.G. Knowles, Nucl. Phys. B 310 (1988) 571;

G. Marchesini et al., Comput. Phys. Commun. 67 (1992) 465 .
[22] GEANT Version 3.13 (September 1989) see R. Brun et al., GEANT 3, CERN DD/EE/84-1 (revised) (September 1987);

to simulate hadronic interactions the program GHEISHA is used,

see H. Fesefeldt, RWTH Aachen report PITHA 85/02 (1985).

[23] JADE Collab., W. Bartel et al., Phys. Lett. B 91 (1980) 142; B 115 (1982) 211.

[24] N. Brown and W.J. Stirling, Phys. Lett. B 252 (1990) 657.

[25] V. Blobel, DESY report 84-118 (1984); RUN General program for regularized unfolding (1984).

[26] R.K. Ellis, D.A. Ross and A.E. Terrano, Nucl. Phys. B 178 (1981) 421 .

[27] Z. Kunszt and P. Nason, in: Z Physics at LEP 1, CERN report CERN-89-08, Vol.I., p. 373.

[28] M. Aguilar-Benitez et al., Phys. Lett. B 239 (1990) 1.

[29] L3 Collab., B. Adeva et al., Phys. Lett. B 241 (1990) 416; B 261 (1991) 177.

[30] B.L. loffe, Phys. Lett. B 78 (1978) 277.

[31] ALEPH Collab., D. Decamp et al., Measurement of $\alpha_{S}$ in hadronic $Z$ decays using all-orders resummed predictions, CERN preprint CERN-PPE/92-33.

[32] L3 Collab., B. Adeva et al., Z. Phys. C 51 (1991) 179. 